\title{
CALCULATION OF TEMPERATURE DISTRIBUTION AND THERMO-OPTICAL EFFECTS IN DOUBLE-END-PUMPED SLAB LASER
}

The temperature distribution and thermo-optical effects in a double-end-pumped slab laser are investigated analytically. The theoretical model is given by considering heat generation on both sides of an active medium due to pumping. With account for the pump beam divergence and the heat load, the heat conduction equation is solved, and the temperature distribution and thermal effects, such as thermal lensing and thermal stress, are obtained. The results are applied to a typical Nd:YVO $\mathrm{O}_{4}$ laser crystal slab and discussed.

Keywords: slab lasers, double-end-pumping, thermo-optical effects.

Introduction. Diode end-pumped solid-state lasers have received considerable attention due to their high efficiency, compact arrangement, and good beam quality. Their outstanding properties lead to wide applications, from industrial to medical ones. The thermal effects, such as thermal lensing and thermal stress, can influence the performance and quality of these lasers, especially in a high power regime $[1,2]$. These effects arising from pump heat loading influence the optical behavior of the laser and, consequently, beam quality. In a high power regime, these thermal effects can influence the resonator stability position on the plane of the stability curve and cannot be neglected in designing laser resonators. Reducing and compensating the thermal effects are of significance in the high power laser regime. Many ways have been proposed to attain this goal. Among them, using composite crystals [3], designing resonators of variable configuration [4,5], and double-end-pumping scheme [6] are the best known. Rectangular crystals of special slab shape show great potential for thermal effect reduction. A large coolant surface [7-9] allows them to remove more heat than using crystals of other shapes.

In this paper, the temperature distribution and thermal effects, such as thermal lensing, thermal induced stress, and stress induced change in the refractive index, for the double-end-pumped slab are investigated analytically. Explicit relations are obtained to calculate the mentioned effects, and a typical $\mathrm{Nd}: \mathrm{YVO}_{4}$ laser crystal is used to demonstrate the results. The results are compared with those for a single-end-pumped configuration.

Temperature Distribution. The geometry of the double-end-pumped slab crystal is shown in Fig. 1. The crystal of dimensions $a \times c \times L(c<L$ and $c<a)$ is pumped from two end faces $(z=0, z=L)$ by two multi-bar diode stacks. The optical axis of the crystal is directed along the $z$ direction and the direction of the output laser beam is the same as that of the pump beam.

The general steady-state heat conduction equation for an isotropic medium in the Cartesian coordinate system is

$$
\frac{\partial^{2} T(x, y, z)}{\partial x^{2}}+\frac{\partial^{2} T(x, y, z)}{\partial y^{2}}+\frac{\partial^{2} T(x, y, z)}{\partial z^{2}}=-\frac{Q(x, y, z)}{k},
$$

where $Q(x, y, z)$ is the heat generation per unit volume. The pump beam can be assumed to be of an inhomogeneous intensity with the Gaussian profile along the $y$ axis and of homogenous one along the $x$ direction. Considering the propagation along the $z$ axis, we write the heat power density as

$$
Q(y, z)=Q_{1} \exp \left(-\frac{y^{2}}{\omega_{1}^{2}(z)}\right) \exp (-\alpha z)+Q_{\mathrm{r}} \exp \left(-\frac{y^{2}}{\omega_{\mathrm{r}}^{2}(z)}\right) \exp (-\alpha(L-z)),|y|<b / 2 ;
$$

Physics Department, Bilkent University, Cankaya, Ankara 06800, Turkey; email: pelahi@ fen.bilkent.edu.tr. Published in Inzhenerno-Fizicheskii Zhurnal, Vol. 84, No. 6, pp. 1142-1147, November-December, 2011. Original article submitted August 22, 2010; revision submitted March 1, 2011. 

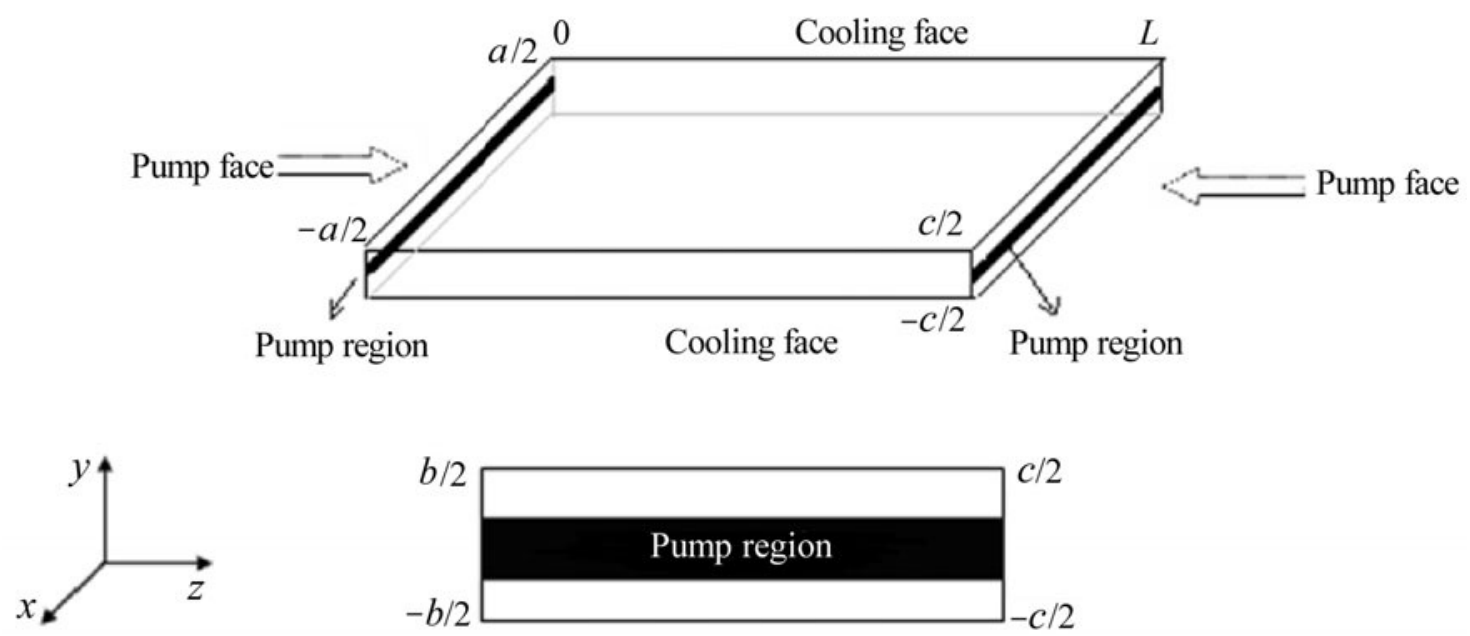

Fig. 1. Schematic diagram of the double-end-pumped slab crystal and pump region.

$$
Q(y, z)=0, \quad b / 2<|y|<c / 2
$$

where the left and right heat power densities can be calculated in the following way:

$$
\begin{gathered}
Q_{1}=\frac{\eta P_{1}}{a \int_{-b / 20}^{b / 2} \int^{L} \exp \left(-\frac{y^{2}}{\omega_{1}^{2}(z)}\right) \exp (-\alpha z) d y d z}, \\
Q_{\mathrm{r}}=\frac{\eta P_{\mathrm{r}}}{a \int_{-b / 20}^{b / 2} \int^{L} \exp \left(-\frac{y^{2}}{\omega_{\mathrm{r}}^{2}(z)}\right) \exp (-\alpha(L-z)) d y d z} .
\end{gathered}
$$

In the above equations the heat generation efficiency is expressed as $\eta=1-\lambda_{p} / \lambda_{\text {laser }}$, and the left and right pump spot sizes can be written as

$$
\omega_{1}(z)=\omega_{0} \sqrt{1+\left[\frac{M^{2} \lambda_{\mathrm{p}} z}{n \pi \omega_{0}^{2}}\right]^{2}}, \quad \omega_{\mathrm{r}}(z)=\omega_{0} \sqrt{1+\left[\frac{M^{2} \lambda_{\mathrm{p}}(L-z)}{n \pi \omega_{0}^{2}}\right]^{2}},
$$

where $M^{2}$ is the measure of deviation from the Gaussian beam.

Analytical Solution. Because of the strong cooling system at $y= \pm c / 2$, the cooling coefficient in the $y$ direction is much higher than those in other directions. Then we can assume that the heat flow is directed only along the $y$ axis. Therefore, the general heat conduction equation (1) can be simplified to a one-dimension case:

$$
\frac{\partial^{2} T(x, y, z)}{\partial y^{2}}=\left\{\begin{array}{cl}
-\frac{Q(y, z)}{k}, & \text { pump region } \\
0, & \text { outside }
\end{array}\right.
$$

The following boundary conditions are used for the temperature:

$$
\left.\frac{\partial T_{1}}{\partial y}\right|_{y=0}=0
$$



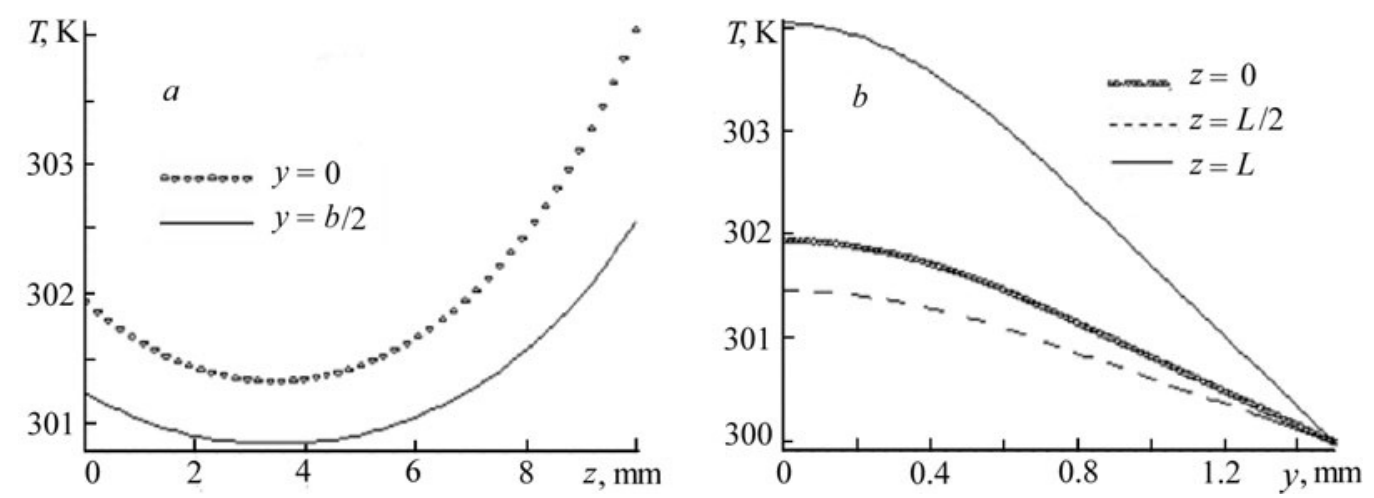

Fig. 2. Temperature distributions in the double-end-pumped slab with $P_{1}=$ $3 \mathrm{~W}, P_{\mathrm{r}}=7 \mathrm{~W}$ along the $z$ (a) and $y(\mathrm{~b})$ axes.

which implies the existence of a temperature maximum at the center;

$$
\left.T_{1}\right|_{y=b / 2}=T_{2},\left.\frac{\partial T_{1}}{\partial y}\right|_{y=b / 2}=\left.\frac{\partial T_{2}}{\partial y}\right|_{y=b / 2},
$$

i.e., conditions indicating that the temperature and its derivative are continuous on the region boundary;

$$
\left.T_{2}\right|_{y=c / 2}=T_{\mathrm{c}},
$$

which is evidence that the constant surface temperature is equal to the coolant temperature. Under the above boundary conditions, the following solutions are obtained for the temperature distribution in the pumped region and outside:

$$
\begin{aligned}
& T_{1}=-\frac{\eta \alpha}{k}\left[\frac{I_{1} \sqrt{\pi} \omega_{1}^{2}(z)}{2 \exp (\alpha z)}\left(\frac{y}{\omega_{1}(z)} \operatorname{erf}\left(\frac{y}{\omega_{1}(z)}\right)+\frac{1}{\sqrt{\pi}} \exp \left(-\frac{y^{2}}{\omega_{1}^{2}(z)}\right)\right)\right. \\
& \left.+\frac{I_{\mathrm{r}} \exp (\alpha z) \sqrt{\pi} \omega_{\mathrm{r}}^{2}(z)}{2 \exp (\alpha L)}\left(\frac{y}{\omega_{\mathrm{r}}(z)} \operatorname{erf}\left(\frac{y}{\omega_{\mathrm{r}}(z)}\right)+\frac{1}{\sqrt{\pi}} \exp \left(-\frac{y^{2}}{\omega_{\mathrm{r}}^{2}(z)}\right)\right)\right] \\
& +\frac{\eta \alpha}{k}\left[\frac{I_{1} \sqrt{\pi} \omega_{1}^{2}(z)}{2 \exp (\alpha z)}\left(\frac{b}{2 \omega_{1}(z)} \operatorname{erf}\left(\frac{b}{2 \omega_{1}(z)}\right)+\frac{1}{\sqrt{\pi}} \exp \left(-\frac{b^{2}}{4 \omega_{1}^{2}(z)}\right)\right)\right. \\
& \left.+\frac{I_{\mathrm{r}} \exp (\alpha z) \sqrt{\pi} \omega_{\mathrm{r}}^{2}(z)}{2 \exp (\alpha L)}\left(\frac{b}{2 \omega_{\mathrm{r}}(z)} \operatorname{erf}\left(\frac{b}{2 \omega_{\mathrm{r}}(z)}\right)+\frac{1}{\sqrt{\pi}} \exp \left(-\frac{b^{2}}{4 \omega_{\mathrm{r}}^{2}(z)}\right)\right)\right] \\
& -\frac{\eta \alpha}{k}\left[\frac{I_{1} \sqrt{\pi} \omega_{1}(z)}{2 \exp (\alpha z)} \operatorname{erf}\left(\frac{b}{2 \omega_{1}(z)}\right)+\frac{I_{\mathrm{r}} \exp (\alpha z) \sqrt{\pi} \omega_{\mathrm{r}}(z)}{2 \exp (\alpha L)} \operatorname{erf}\left(\frac{b}{2 \omega_{\mathrm{r}}(z)}\right)\right]\left(\frac{b}{2}-\frac{c}{2}\right)+T_{\mathrm{c}}, \\
& T_{2}=-\frac{\eta \alpha}{k}\left[\frac{I_{1} \sqrt{\pi} \omega_{1}(z)}{2 \exp (\alpha z)} \operatorname{erf}\left(\frac{b}{2 \omega_{1}(z)}\right)+\frac{I_{\mathrm{r}} \exp (\alpha z) \sqrt{\pi} \omega_{\mathrm{r}}(z)}{2 \exp (\alpha L)}\right. \\
& \left.\times \operatorname{erf}\left(\frac{b}{2 \omega_{\mathrm{r}}(z)}\right)\right] y-\frac{c}{2} \frac{\eta \alpha}{k}\left[\frac{I_{1} \sqrt{\pi} \omega_{1}(z)}{2 \exp (\alpha z)} \operatorname{erf}\left(\frac{b}{2 \omega_{1}(z)}\right)+\frac{I_{\mathrm{r}} \exp (\alpha z) \sqrt{\pi} \omega_{\mathrm{r}}(z)}{2 \exp (\alpha L)} \operatorname{erf}\left(\frac{b}{2 \omega_{\mathrm{r}}(z)}\right)\right]+T_{\mathrm{c}} .
\end{aligned}
$$



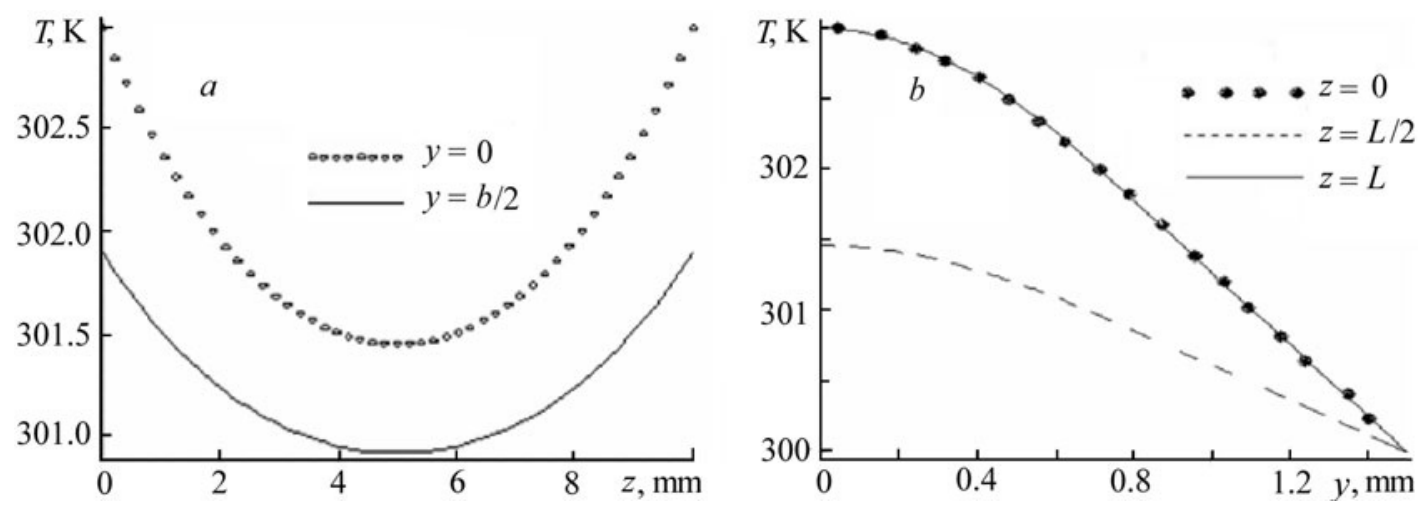

Fig. 3. Same as in Fig. 2 with $P_{1}=P_{\mathrm{r}}=5 \mathrm{~W}$.
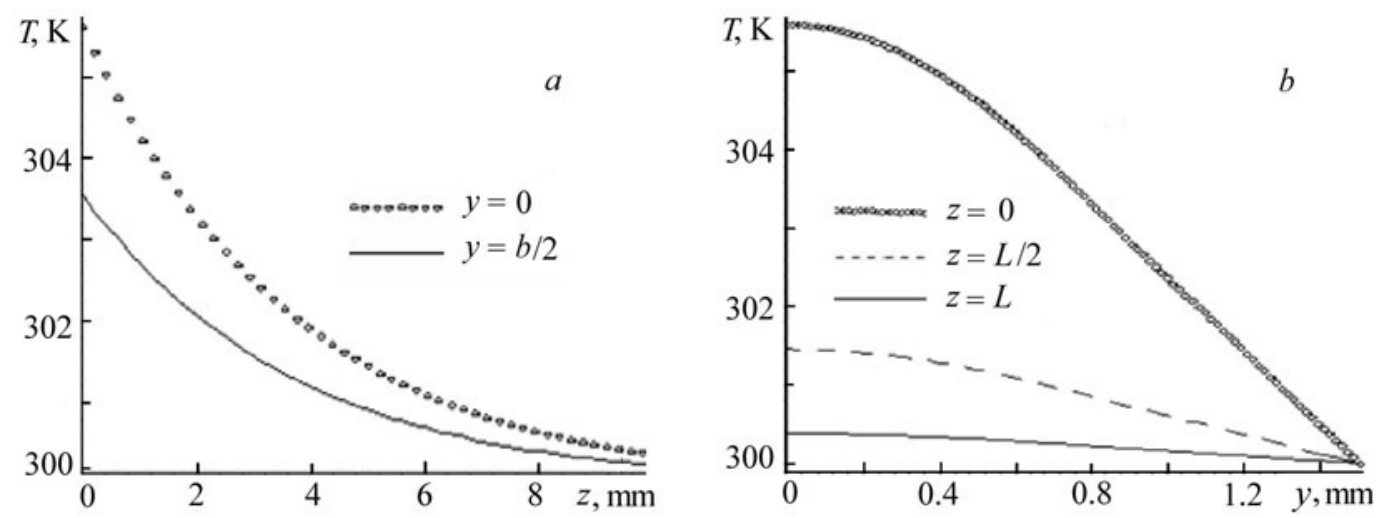

Fig. 4. Temperature distributions in the single-end-pumped slab with $P_{1}=10$

$\mathrm{W}$ along the $z$ (a) and $y$ (b) axes.
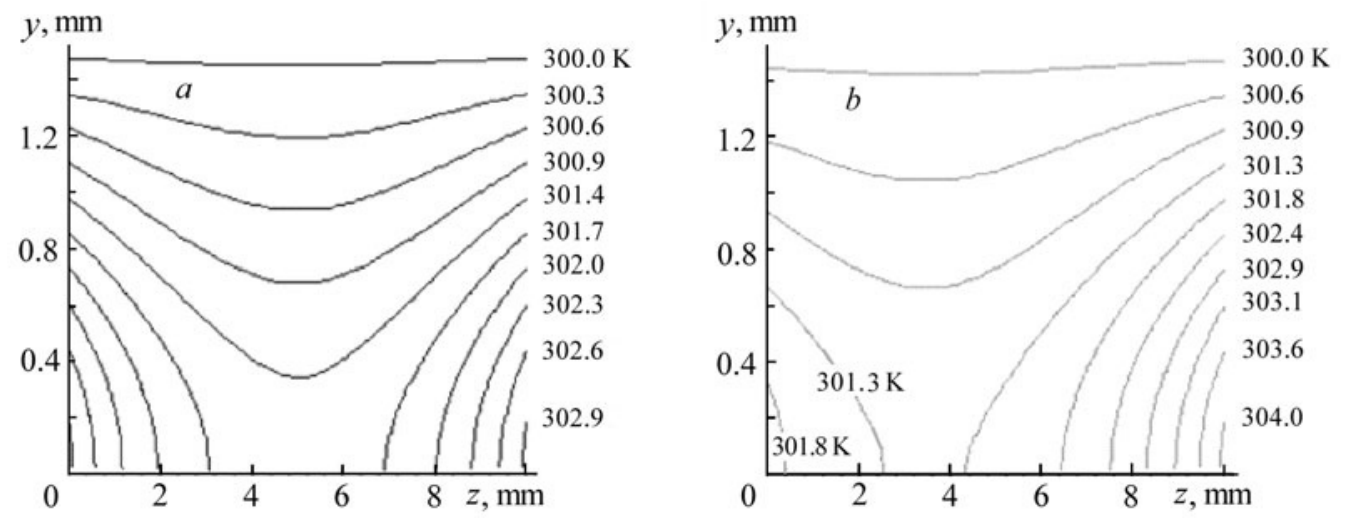

Fig. 5. Isotherms for the double-end-pumped slab with $P_{1}=3 \mathrm{~W}, P_{\mathrm{r}}=7 \mathrm{~W}$ (a) and $P_{1}==P_{\mathrm{r}}=5 \mathrm{~W}(\mathrm{~b})$.

Results and Discussion. The obtained results have been illustrated by the example of a vanadate crystal. Here, we considered a Nd: $\mathrm{YVO}_{4}$ slab with $L=10 \mathrm{~mm}, c=3 \mathrm{~mm}, a=10 \mathrm{~mm}$, and $b=1.5 \mathrm{~mm}$ which was pumped from both sides by diode lasers with a wavelength of $806 \mathrm{~nm}$. At first, we considered the case with $P_{1}=3 \mathrm{~W}$ and $P_{\mathrm{r}}$ $=7 \mathrm{~W}$. The minimum spot size was $\omega_{0}=0.75 \mathrm{~mm}$. Figure 2 shows the $2 \mathrm{D}$ graphs of the temperature distributions along the $z$ axis for two values of $y$ and along the $y$ axis for three values of $z$. As seen from the figure, the maximum temperature rise is about 304 and $302 \mathrm{~K}$ at the center of the right and left faces, respectively.

In Fig. 3, the temperature distributions in the slab are plotted versus $z$ and $y$ for equal pump powers $P_{1}=P_{\mathrm{r}}$ $=5 \mathrm{~W}$. As shown in this figure, the maximum temperature is about $303 \mathrm{~K}$, which is less than that in the previous 


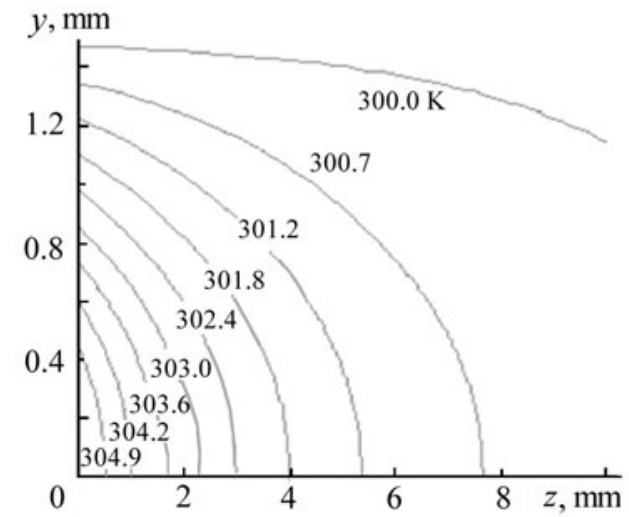

Fig. 6. Isotherms for the single-end-pumped slab with $P_{1}=5 \mathrm{~W}$.

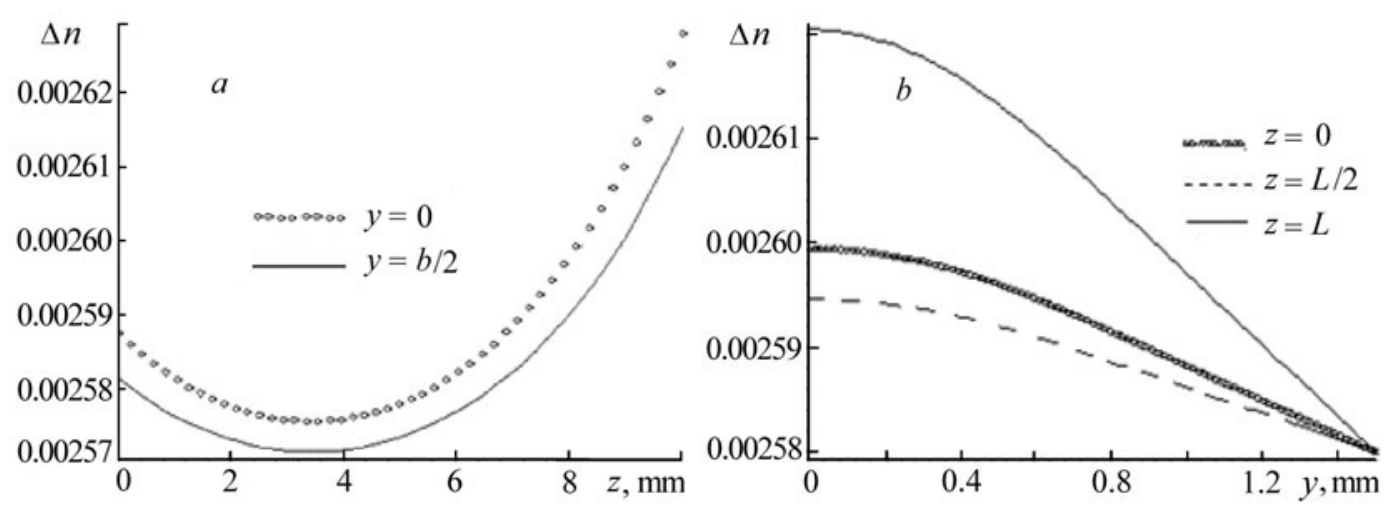

Fig. 7. Change in the refractive index of the double-end-pumped slab with $P_{1}$ $=3 \mathrm{~W}, P_{\mathrm{r}}=7 \mathrm{~W}$ along the $z$ (a) and $y(\mathrm{~b})$ axes.
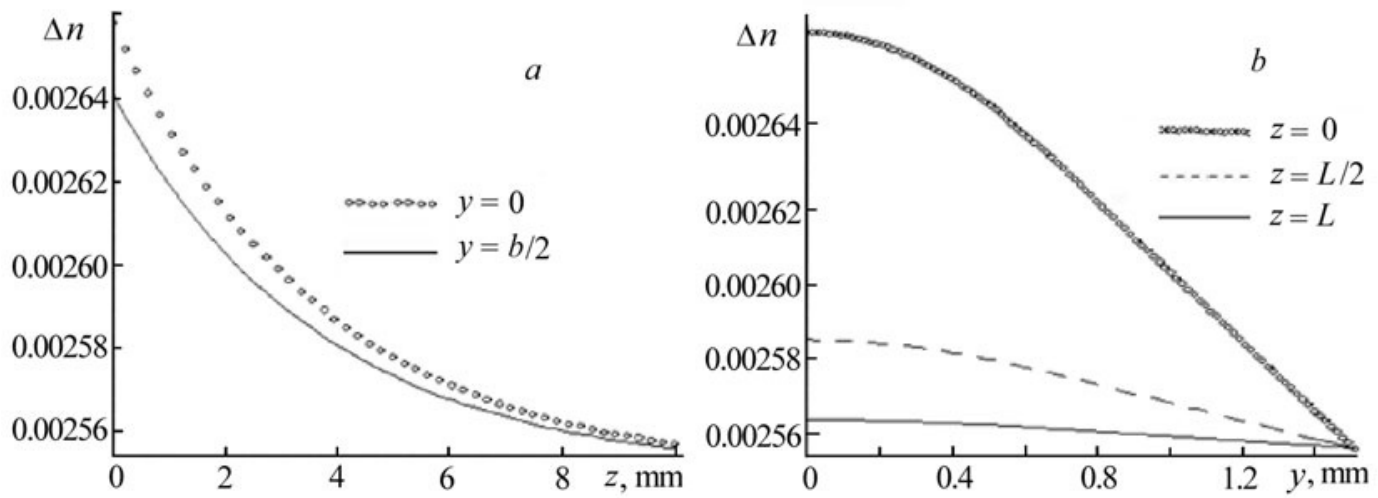

Fig. 8. Change in the refractive index of the single-end-pumped slab with $P_{1}=$ $10 \mathrm{~W}$ along the $z$ (a) and $y$ (b) axes.

configuration. Figure 4 gives the temperature distributions for the single-pumped configuration with $P_{1}=10 \mathrm{~W}$ from whence the temperature increase relative to the double pumping can be seen. Figures 5 and 6 present isotherms for the double-end-pumped slab with different (Fig. 5a) and equal (Fig. 5b) pumping as well as for single-end-pumped one (Fig. 6).

Thermal Effects Analysis. Thermal Lensing. The important influence of the inhomogenous temperature distribution is the thermal lensing which generally signifies a variation of the refractive index with temperature. The temperature-dependent change in the refractive index can be expressed according to [10] as 

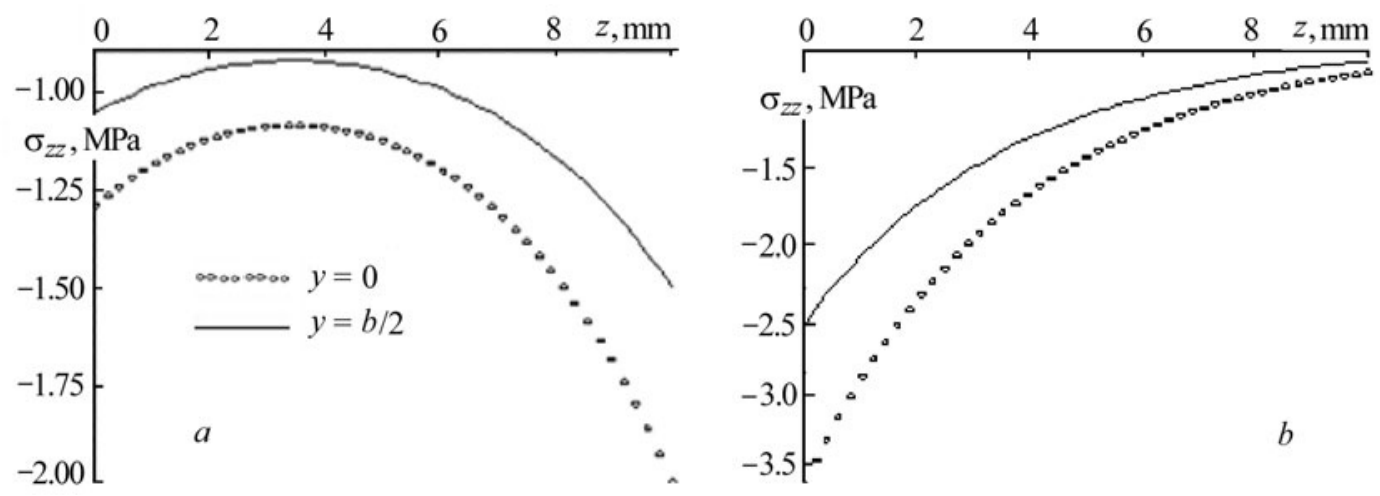

Fig. 9. Change in $\sigma_{z z}$ vs. $z$ for the double-end-pumped slab with $P_{1}=3 \mathrm{~W}, P_{\mathrm{r}}$ $=7 \mathrm{~W}$ (a) and for the single-end-pumped slab with $P_{1}=10 \mathrm{~W}$ (b).

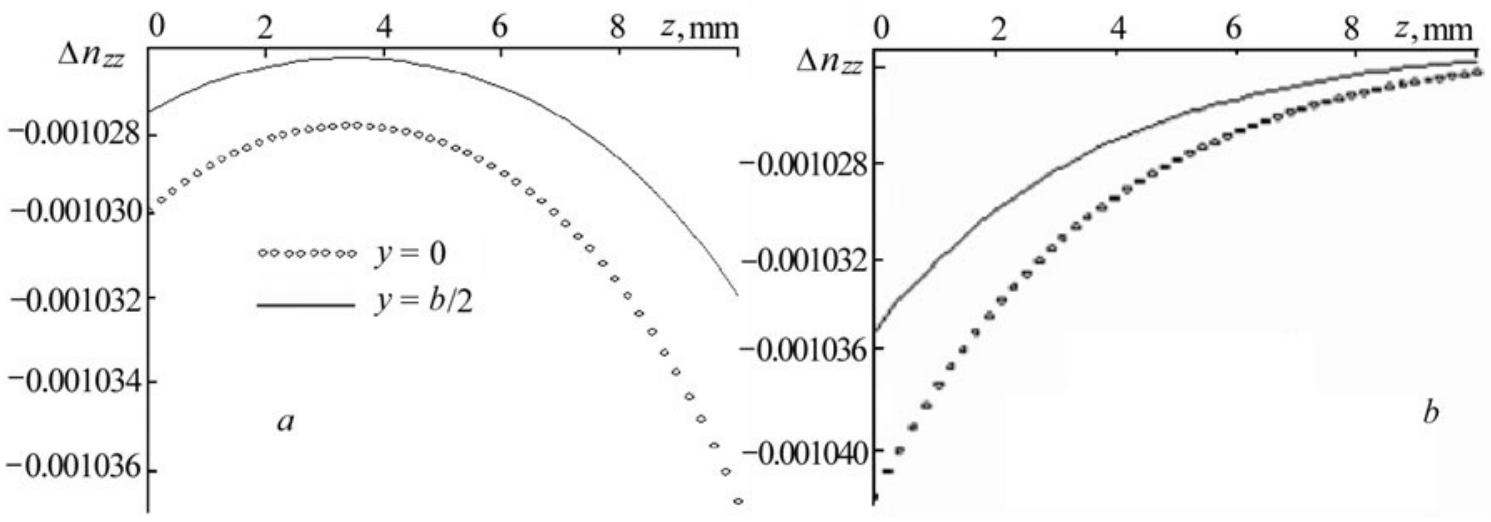

Fig. 10. Same as in Fig. 9 but for change in $\Delta n_{z z}$.

$$
\Delta n=\left[T(x, y, z)-T_{0}\right] \frac{d n}{d T}
$$

For the $\mathrm{Nd}: \mathrm{YVO}_{4}$ slab laser, variations of the refractive index for a sample along both the $z$ and $y$ directions in the double- and single-end-pumped slabs are shown in Figs. 7 and 8, respectively.

Thermal Stress. In the absence of the thermal loading, the slab is assumed to be stress-free, so that it is not exposed to external forces. The induced thermal stress distributions are generated by the temperature gradients. As discussed in [11], it can be shown that the stress components can be found from

$$
\begin{aligned}
& \sigma_{x x}=\frac{\left(\alpha_{y}^{\prime}+v \alpha_{z}^{\prime}\right) \mathrm{E} T(y, z)}{v^{2}-1}, \\
& \sigma_{z z}=\frac{\left(v \alpha_{y}^{\prime}+\alpha_{z}^{\prime}\right) \mathrm{E} T(y, z)}{v^{2}-1},
\end{aligned}
$$

where $\mathrm{E}=1.33 \cdot 10^{11}, v=0.33, \alpha_{z}^{\prime}=11.37 \cdot 10^{-6} \mathrm{~K}^{-1}, \alpha_{y}^{\prime}=4.43 \cdot 10^{-6} \mathrm{~K}^{-1}$. As shown in [7], $\sigma_{y y}=0$ everywhere. The induced thermal stress distributions along the $z$ direction for the double- and single-end-pumped slabs are shown in Fig. 9. We presented the figures only for $\sigma_{z z}(z)$, but the dependences $\sigma_{x x}(z)$ are the same, differing only by lower absolute values. It is seen that the stress for the single pumping is greater. Thus, the double pumped configurations are more stress-safe.

The stress-dependent variation of the refractive index can be calculated [11] as 


$$
\begin{aligned}
& \Delta n_{x x}=-B_{\perp} \sigma_{z z}-B_{\|} \sigma_{x x}, \\
& \Delta n_{y y}=-B_{\perp}\left(\sigma_{x x}+\sigma_{y y}\right), \\
& \Delta n_{z z}=-B_{\perp} \sigma_{x x}-B_{\|} \sigma_{z z},
\end{aligned}
$$

where for the Nd: $\mathrm{YVO}_{4}$ crystal $B \|=-2.2 \cdot 10^{-12}, B_{\perp}=0.42 \cdot 10^{-12}$. The stress induced variations of the refractive index $\Delta n_{z z}$ along the $z$ axis for both double- and single-end-pump are shown in Fig. 10. The omitted dependences for $\Delta n_{x x}$ are characterized by analogous behavior with higher absolute values.

Conclusions. The temperature distribution and thermo-optical effects of the actual high-power double-endpumped Nd: $\mathrm{YVO}_{4}$ laser slab have been studied. The analytical results for such a typical slab have been compared with those for the single-end-pumped configuration. The double-end-pumped slab laser with equal pump powers at both faces is shown to have a lower temperature and, consequently, lesser thermo-optical effects as compared to other pumping. Thus, a high power regime with an equal-end-pumped configuration is suggested.

\section{NOTATION}

$a, c, L$, crystal dimensions, $\mathrm{m} ; b$, pump region size, $\mathrm{m} ; B_{\|}, B_{\perp}$, stress optical tensor components, $\mathrm{Pa}^{-1}$; E, Young's modulus, $\mathrm{Pa} ; \mathrm{I}$, input power intensity, $\mathrm{W} / \mathrm{m}^{2} ; k$, thermal conductivity, $\mathrm{W} /\left(\mathrm{m}^{2} \cdot \mathrm{K}\right) ; M^{2}$, measure of deviation from the Gaussian beam; $n$, refractive index of crystal at pump wavelength; $P$, input power, W; $Q$, heat power density, $\mathrm{W} / \mathrm{m}^{3} ; T$, temperature, $\mathrm{K} ; T_{\mathrm{c}}$, coolant temperature, $\mathrm{K} ; T_{1}$ and $T_{2}$, temperatures in the pumped region and outside, respectively, $\mathrm{K} ; x, y, z$, coordinates, $\mathrm{m} ; \alpha$, absorption coefficient, $\mathrm{m}^{-1} ; \alpha^{\prime}$, thermal expansion coefficient, $\mathrm{K}^{-1} ; \eta$, quantum efficiency; $\lambda$, wavelength, $\mathrm{nm} ; \mathrm{v}$, Poisson ratio; $\sigma$, stress, Pa; $\omega$, pump spot size, $\mathrm{m}$; $\omega_{0}$, beam waist size, m. Indices: c, coolant; l, left; p, pumping; $r$, right.

\section{REFERENCES}

1. Fuyan Lu, Mali Gong, Haizong Xue, Qiang Liu, and Wupeng Gong, Analysis of the temperature distribution and thermal effects in corner-pumped slab lasers, Opt. Lasers Eng., 45, $43-48$ (2007).

2. H. Nadgaran and P. Elahi, The overall phase shift and lens effect calculation using Gaussian boundary condition and paraxial ray approximation for an end-pumped solid-state laser, Pramana - J. Phys., 66, No. 3, 513 (2006).

3. M. P. MacDonald, Th. Graf, J. E. Balmer, and H. P. Weber, Reducing thermal lensing in diode-pumped laser rods, Opt. Commun., 78, 383-393 (2000).

4. Th. Graf, E. Wyss, M. Roth, and H. P. Weber, Laser resonator with balanced thermal lenses, Opt. Commun., 190, 327-331 (2001).

5. Yao Ai-Yun, Hou Wei, Li Hui-Qing, Bi Yong, Lin Xue-Chun, Geng Ai-Cong, Kong Yu-Peng, Cui Da-Fu, and $\mathrm{Xu} \mathrm{Zu}$-Yan, Reducing thermal effect in end-diode-pumped laser crystal by using a novel resonator, Chin. Phys. Lett, 22, No. 3, 607-610 (2005).

6. H. Ogilvy, M. J. Withford, P. Dekker, and J. A. Piper, Efficient diode double-end-pumped Nd:YVO4 laser operating at $1342 \mathrm{~nm}$, Opt. Express, 11, No. 19, 2411-2415 (2003).

7. J. M. Eggleston, T. J. Kane, K. Kuhn, J. Unternahrer, and R. L. Byer, The slab geometry laser — I: Theory, IEEE J. Quantum Electron., 20, 289-301 (1984).

8. T. Kane, J. Eggleston, and R. Byer, The slab geometry laser - II: Thermal effects in a finite slab, IEEE J. Quantum Electron., 21, 1195-2101 (1985).

9. P. Elahi and S. Morshedi, The double-end-pumped cubic Nd:YVO 4 lasers: the temperature distribution and thermal stress, Pramana - J. Phys., 74, No. 1, 67-74 (2010).

10. W. Koechner, Solid-State Laser Engineering, Sixth ed., Springer Verlag, Berlin (2006).

11. Zhe Ma, Daijun Li, Jiancum Gao, Nianle We, and Keming Du, Thermal effects of the diode end-pumped Nd:YVO4 slab, Opt. Commun., 275, 179-185 (2007). 